\title{
An open-label, randomized, phase 3 study of the efficacy and safety of antithrombin gamma in patients with sepsis-induced disseminated intravascular coagulation syndrome
}

\author{
Shigeatsu Endo ${ }^{1 *}$ (D) Ryutaro Shimazaki ${ }^{2}$ and the Antithrombin Gamma Study Group
}

\begin{abstract}
Background: A recombinant form of antithrombin (AT), called AT gamma, is being developed as an alternative to AT derived from human plasma. To compare the efficacy and safety of AT gamma to plasma-derived AT (pAT), we conducted a randomized, open-label, multicenter trial in patients with sepsis-induced disseminated intravascular coagulation (DIC).

Methods: Eligible patients, recruited at 30 clinical sites, had been diagnosed with sepsis-induced DIC (by the Japanese Association for Acute Medicine [JAAM] DIC criteria) and AT activity at 70\% or below. Patients were randomized 1:1 to either $36 \mathrm{IU} / \mathrm{kg} /$ day AT gamma $(n=110)$ or $30 \mathrm{IU} / \mathrm{kg} /$ day PAT $(n=112)$, both administered intravenously for 5 days. The primary endpoint was recovery from DIC at day 6 or early study withdrawal. DIC recovery was defined as a DIC score of less than four. Secondary endpoints were DIC score, outcome on day 28, sequential organ failure assessment score, acute physiology and chronic health evaluation II score (APACHE II), and plasma AT activity. Adverse events and adverse drug reactions were recorded using MedDRA/J version 16.0.
\end{abstract}

Results: Baseline patient demographics and clinical features were similar in the two treatment groups. On day 6 (or at withdrawal), DIC recovery had occurred in 62 of 110 (56.4\%; 95\% confidence interval, 46.6-65.8\%) patients in the AT gamma group and 59 of 112 (52.7\%; 95\% confidence interval, 43.0-62.2\%) patients in the pAT group. In both treatment groups, DIC recovery rate values tended to be higher when stratified by baseline AT activity rates. All changes in other secondary endpoints were similar in both treatment groups. Safety was also similar in the two treatment groups. Adverse events occurred in 89 of 108 (82.4\%) patients in the AT gamma group and 99 of 113 (87.6\%) patients in the PAT group.

Conclusions: Safety and efficacy were similar for $36 \mathrm{IU} / \mathrm{kg} /$ day AT gamma and $30 \mathrm{IU} / \mathrm{kg} /$ day PAT. These results confirm that AT gamma is an excellent alternative to PAT for improving outcomes for patients with DIC.

Trial registration: ClinicalTrials.gov identifier: NCT01384903; June 2011.

Keywords: Recombinant form of human AT, AT gamma, DIC, DIC recovery, Survival, JAAM DIC criteria, AT activity, Potelligent ${ }^{\oplus}$

\footnotetext{
* Correspondence: endo@yu-ai.net

Shigeatsu Endo (endo@yu-ai.net), the corresponding author, is the

representative and coordinator of the Antithrombin Gamma Study Group.

${ }^{1}$ Morioka Yuai Hospital, Nagai 12-10, Morioka, Iwate 020-0834, Japan

Full list of author information is available at the end of the article
}

(c) The Author(s). 2018 Open Access This article is distributed under the terms of the Creative Commons Attribution 4.0 International License (http://creativecommons.org/licenses/by/4.0/), which permits unrestricted use, distribution, and reproduction in any medium, provided you give appropriate credit to the original author(s) and the source, provide a link to the Creative Commons license, and indicate if changes were made. The Creative Commons Public Domain Dedication waiver (http://creativecommons.org/publicdomain/zero/1.0/) applies to the data made available in this article, unless otherwise stated. 


\section{Background}

Antithrombin (AT), a major coagulation inhibitor in humans, is a single-chain glycoprotein with 432 amino acids and a molecular weight of approximately $58 \mathrm{kDa}$ $[1,2]$. AT binds and traps thrombin and other coagulation factors, including factors IX, X, XI, and XII [3, 4]. AT's activity is enhanced 1000-fold by heparin [5].

Functional AT levels are significantly reduced in patients with congenital AT deficiency, and affected individuals are susceptible to clotting from a young age. Acquired AT deficiency can result from either decreased production or increased degradation of AT and is often associated with disseminated intravascular coagulation (DIC) [6], a life-threatening condition characterized by activated coagulation pathways, decreased anticoagulant activity, and altered activity of fibrinolytic pathways [6, 7]. In patients with DIC, numerous microthrombi form in the blood; however, patients may also experience excessive bleeding if clotting factors become depleted. DIC is typically secondary to other conditions such as sepsis and trauma [6].

Although the primary goal for treatment of patients with DIC is to address the underlying cause, various forms of AT have been developed that can be used to reduce the damage caused by DIC and improve survival. AT can also be used therapeutically to prevent clots in people with inherited AT deficiency, primarily during postoperative and perinatal time periods [8]. A large multinational randomized trial (KyberSept) found that AT treatment did not improve outcomes for patients with sepsis [9]. However, a reanalysis of the data focusing on the patients with DIC found a reduction in mortality in those patients treated with AT [10]. A systematic review of 32 trials confirmed this result [11]. A recent large, multicenter, retrospective study of 1784 patients diagnosed with DIC in Japan found that AT supplementation was associated with reduced mortality [12]. AT is widely used to treat DIC in Japan $[8,13]$. The importance of $\mathrm{AT}$ and recommendations for its use are continually being evaluated as new data emerge.

A recombinant form of AT, called AT alfa (ATryn ${ }^{\circ}$ ), has been developed to provide a stable source of pathogen-free AT. Recombinant AT alfa, purified from the milk of goats that have been engineered to express human AT, is approved in the European Union and the USA for prevention of venous thromboembolism in patients with congenital AT deficiency during surgery [14]. However, the glycosylation profile of AT alfa differs from that of native AT, resulting in a dramatically reduced half-life [15]. To maintain adequate AT alfa levels, the drug must be administered by intravenous injection for $24 \mathrm{~h}$.

AT gamma (KW-3357) is a new form of recombinant AT that has a sugar chain structure similar to that of native human AT. The drug was developed using Potelligent $^{\circ}$ technology, which relies on a host Chinese hamster ovary cell line that produces fucose-free recombinant proteins [16]. Native AT does not contain any fucose moieties, and because of this structural similarity, AT gamma has pharmacokinetic and biological activities similar to those of native AT.

AT gamma is being developed as an alternative to plasma-derived AT (pAT) products. Like pAT, AT gamma has both $\alpha$ and $\beta$ isoforms (the $\alpha$ isoform is the dominant form, with an oligosaccharide occupying each of its four glycosylation sites, while the $\beta$ isoform, representing $5-15 \%$ of the total AT found in human plasma [15], is a minor form that lacks glycosylation at one of these sites). For AT gamma, these isoforms are present in similar ratios to those seen for pAT (data on file, Kyowa Hakko Kirin Co., Ltd., Tokyo, Japan). Recombinant AT alfa also has both of these isoforms, and although the ratio has not been clearly defined, one report has suggested that the $\alpha$ isoform constitutes more than $80 \%$ [15]. Aside from the difference in the heterogeneity of the sugar chains, the main types of oligosaccharides (complex-type nonfucosylated N-linked oligosaccharides) in AT gamma and pAT are the same; however, AT gamma does not have any of the risks of infection that pAT has and can be stably produced without dependence on human plasma donors [15].

In a study in healthy male volunteers, AT gamma (72 $\mathrm{IU} / \mathrm{kg}$ ) was found to be bioequivalent to pAT $(60 \mathrm{IU} / \mathrm{kg}$ ) (data on file; Kyowa Hakko Kirin Co., Ltd., Tokyo, Japan). This multicenter, open-label, randomized, parallel-group, active-controlled, phase 3 study used doses of $36 \mathrm{IU} / \mathrm{kg}$ and $30 \mathrm{IU} / \mathrm{kg}$ for AT gamma and pAT, respectively. The objective was to evaluate the efficacy and safety of AT gamma in patients with septic DIC.

\section{Methods}

\section{Patients}

This study, conducted at 30 sites, enrolled patients aged $\geq 20$ years with sepsis-induced DIC. Eligible patients met the American College of Chest Physicians/Society of Critical Care Medicine definitions for sepsis, had sepsis-induced DIC (calculated from DIC scores for four categories [systemic inflammatory response syndrome, platelets, prothrombin time (PT) ratio, and fibrinogen degradation product] of the Japanese Association for Acute Medicine (JAAM) DIC criteria [17]), and had AT activity $\leq 70 \%$. The original protocol required patients to have AT activity from 50 to $70 \%$, but the protocol was amended to include all patients with AT activity $\leq 70 \%$ so that the patient population would more accurately reflect the intended clinical use. In addition, the amendment allowed for use of thrombomodulin alfa (Recomodulin $\left.{ }^{\circ}\right)$. The following patients were excluded from the study: patients with drug allergies; patients with serious hepatic dysfunction; patients unlikely to survive long enough to provide efficacy and safety data; patients who were pregnant, breastfeeding, or 
who may have been pregnant; patients who had participated in another clinical study in the previous 4 months; patients who had previously received AT gamma; patients who had received prohibited concomitant medications or therapies between informed consent and enrollment; and patients judged to be ineligible by the investigator.

\section{Study design and treatments}

An enrollment center randomized patients 1:1 to the two groups using a permuted block design (Fig. 1). The study was conducted unblinded. The area under the curve for pAT was 1.2-fold higher than that for AT gamma in the bioequivalence study when using healthy adult Japanese men (data on file; Kyowa Hakko Kirin Co., Ltd., Tokyo, Japan). This was possibly due to the slightly smaller sialic acid content per AT gamma protein than that per pAT protein. Therefore, a 1.2-fold higher dose (72 IU/kg) of AT gamma was considered appropriate. Patients were treated with either $36 \mathrm{IU} / \mathrm{kg} /$ day AT gamma or $30 \mathrm{IU} / \mathrm{kg} /$ day pAT (human anti-thrombin III, Neuart ${ }^{\circ}$ I.V., Japan Blood Products Organization) administered intravenously for 5 days. The daily dose was calculated based on the body weight measured at study enrollment. Heparins were also administered, except in patients for whom concomitant use of heparins could have increased the risk of bleeding. After 5 days of treatment, patients were examined on days 6 and 28. Patients withdrawing from the study before the examination on day 6 were examined promptly after withdrawal.

\section{Efficacy evaluation}

The primary endpoint was the presence or absence of DIC recovery at either day 6 or at withdrawal. DIC recovery was defined as a DIC score of $<4$ calculated according to the JAAM DIC criteria. The secondary endpoints were DIC score, survival of patients on day 28 , sequential organ failure assessment (SOFA) score, acute physiology and chronic health evaluation (APACHE) II score, and plasma
AT activity. AT activity was measured using the factor IIa method at a centralized laboratory (SRL Medisearch). Using this method, excess heparin was added to each AT sample to form an AT-heparin complex, which in turn binds excess thrombin. The AT-heparin complex inactivates the corresponding amount of excess thrombin, and residual thrombin activity was measured to determine the AT activity in the sample.

\section{Safety evaluation}

The safety of AT gamma was also assessed in comparison to pAT. All adverse events (AEs) were coded using MedDRA/J, Version 16.0. Development of anti-AT gamma antibodies was assessed with an immunoassay. AT inhibition was assessed using a chromogenic synthetic substrate assay for AT activity.

\section{Sample size}

The sample size for this study was set large enough that the 95\% confidence interval for the DIC recovery rate for each treatment group could be estimated with an accuracy of $\pm 10 \%$. The DIC recovery rate was assumed to be $50 \%$, which maximizes the confidence interval width. The target sample size was 200 patients, with 100 patients per treatment group.

\section{Statistical methods}

Categorical data were summarized as frequency and percentage, and continuous data were summarized with descriptive statistics (number of patients, mean, standard deviation $[\mathrm{SD}]$, minimum, median, and maximum). The primary evaluation time point was day 6 (or at withdrawal if before day 6). The DIC recovery rate and its 95\% confidence interval (CI) were calculated for each treatment group. In addition, the (adjusted) DIC recovery rate and its $95 \%$ CI were calculated for each treatment group based on the Woolson-Bean method, stratified by the AT activity $(<50 \%, 50-70 \%)$ at study

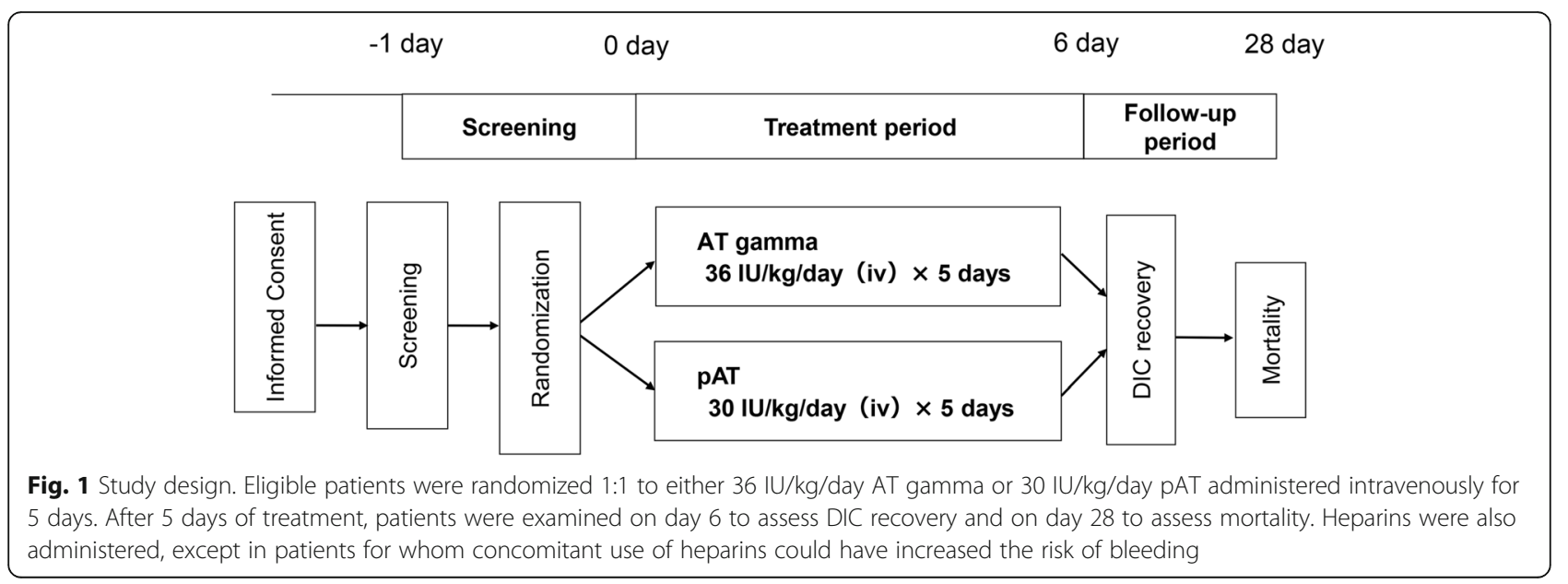


enrollment. For reference, DIC recovery at other time points was also analyzed in the same manner.

The secondary endpoints were analyzed at each time point, and the change from baseline was calculated for each. For outcome on day 28, survival rates were summarized for each treatment group. In addition, the (adjusted) survival rate was calculated for each treatment group based on the Woolson-Bean method stratified by the AT activity $(<50 \%, 50-70 \%)$ at study enrollment. Statistical analysis was performed using SAS 9.2.

\section{Results}

\section{Patients}

In this study, 228 patients were enrolled, and 222 patients were randomized after exclusion of six patients judged to be ineligible for enrollment in the study (Fig. 2). The first informed consent was received in June 2011 and follow-up was completed for all patients by May 2013. Of the 112 patients assigned to the pAT group and 110 patients assigned to the AT gamma group, one patient $(0.9 \%)$ in the AT gamma group withdrew from the study before the start of study treatment. Therefore, 221 patients received study treatment. One hundred and three patients (46.4\%) withdrew from the study after randomization, including $48(43.6 \%)$ in the AT gamma group and 55 (49.1\%) in the pAT group.

Baseline demographic and clinical characteristics were similar in the two groups (Table 1). The mean (SD) age was 70.4 (15.2) in the AT gamma group and 71.0 (15.5) in the pAT group. The mean (SD) DIC score at enrollment was 5.6 (1.2) in the AT gamma group and 5.6 (1.4) in the pAT group. The mean (SD) SOFA scores at baseline were 9.1 (3.3) and 8.9 (3.7) in the AT gamma and pAT groups, respectively. The mean (SD) APACHE II scores at baseline were $18.2(6.5)$ and 18.8 (6.8) in the AT gamma and pAT groups, respectively.
The protocol amendment on AT activity at enrollment did not result in any differences between the groups (Table 1 ). Mean AT activity was $51.5 \%$ in the AT gamma group and $50.9 \%$ in the pAT group. At enrollment, AT activity was less than $50 \%$ in $41(37.3 \%)$ patients in the AT gamma group and $43(38.4 \%)$ patients in the pAT group. AT activity was between 50 and $70 \%$ in 68 patients $(61.8 \%)$ in the AT gamma group and 69 patients $(61.6 \%)$ in the pAT group.

Anticoagulation therapy use was similar in the two groups (Table 1). Thrombomodulin was used by $30.9 \%$ in the AT gamma group and $31.3 \%$ of patients in the pAT group. Heparins were used by $29.1 \%$ in the AT gamma group and $27.7 \%$ of patients in the pAT group.

\section{Primary endpoint analysis}

The primary efficacy analysis was conducted in 222 patients in the intention-to-treat population (110 patients in the AT gamma group and 112 patients in the pAT group). The results are provided in detail in Table 2 . DIC recovery was observed in 62 of 110 (56.4\%, 95\% CI 46.6-65.8\%) patients in the AT gamma group and 59 of 112 (52.7\%, 95\% CI 43.0-62.2\%) patients in the pAT group. For patients with AT activity below $50 \%$ at baseline, the DIC recovery rate was $46.3 \%$ (19 of 41 ) in the AT gamma group and $46.5 \%$ (20 of 43) in the pAT group. For patients with AT activity between 50 and $70 \%$ at baseline, the DIC recovery rate was $61.8 \%$ (42 of 68 ) in the AT gamma group and $56.5 \%$ (39 of 69) in the pAT group.

\section{Secondary endpoint analysis}

All improvements in secondary endpoints with AT gamma were comparable to those seen with pAT (Fig. 3). The change in the DIC score on day 6 (or at withdrawal) was $-2.4 \pm 2.2$ in the AT gamma group and $-2.4 \pm 2.3$ in the pAT group. The survival rate on day 28 was $87.3 \%$

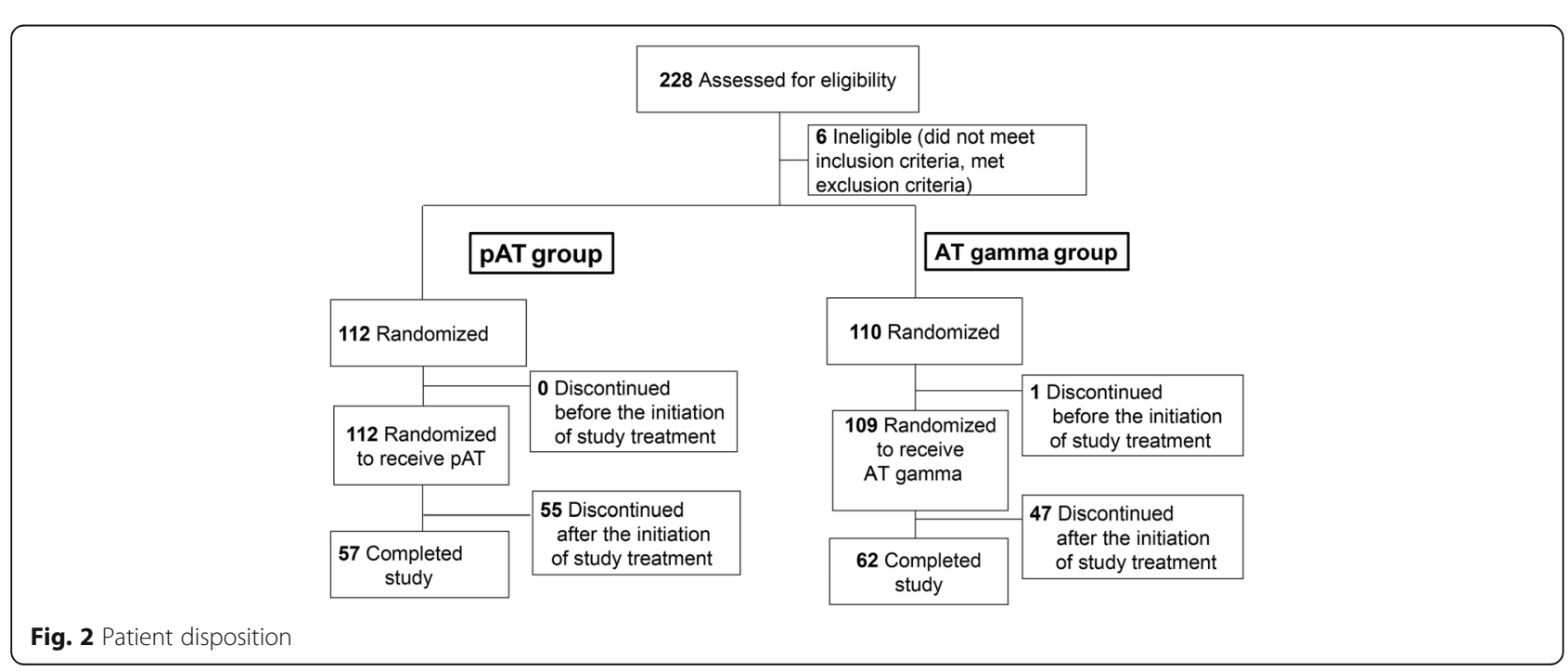


Table 1 Baseline patient characteristics

\begin{tabular}{|c|c|c|c|}
\hline & & $\operatorname{pAT}(N=112)$ & AT gamma $(N=110)$ \\
\hline \multirow[t]{2}{*}{ Sex } & Female & $51(45.5)$ & $51(46.4)$ \\
\hline & Male & $61(54.5)$ & 59 (53.6) \\
\hline \multirow[t]{5}{*}{ Age, years } & Mean \pm SD & $71.0 \pm 15.5$ & $70.4 \pm 15.2$ \\
\hline & $<65$ & $24(21.4)$ & $34(30.9)$ \\
\hline & 65 to $<75$ & $34(30.4)$ & $20(18.2)$ \\
\hline & 75 to $<85$ & $36(32.1)$ & $41(37.3)$ \\
\hline & $\geq 85$ & $18(16.1)$ & $15(13.6)$ \\
\hline \multirow[t]{3}{*}{ Body weight, kg } & Mean \pm SD & $54.3 \pm 12.3$ & $53.8 \pm 14.0$ \\
\hline & $<55$ & $57(50.9)$ & $63(57.3)$ \\
\hline & $\geq 55$ & $55(49.1)$ & $47(42.7)$ \\
\hline \multirow[t]{2}{*}{ Bleeding symptoms at baseline } & Absent & $90(80.4)$ & $93(84.5)$ \\
\hline & Present & $22(19.6)$ & $17(15.5)$ \\
\hline \multirow[t]{3}{*}{ DIC score at enrollment } & Mean \pm SD & $5.6 \pm 1.4$ & $5.6 \pm 1.2$ \\
\hline & $<6$ & $60(53.6)$ & $62(56.4)$ \\
\hline & $\geq 6$ & $52(46.4)$ & $47(42.7)$ \\
\hline \multirow[t]{3}{*}{ SOFA score at enrollment } & Mean \pm SD & $8.9 \pm 3.7$ & $9.1 \pm 3.3$ \\
\hline & $<10$ & $64(57.1)$ & $58(52.7)$ \\
\hline & $\geq 10$ & $47(42.0)$ & $50(45.5)$ \\
\hline \multirow[t]{3}{*}{ APACHE II score at enrollment } & Mean \pm SD & $18.8 \pm 6.8$ & $18.2 \pm 6.5$ \\
\hline & $<20$ & $68(60.7)$ & $61(55.5)$ \\
\hline & $\geq 20$ & $41(36.6)$ & $47(42.7)$ \\
\hline \multirow[t]{3}{*}{ AT activity (\%) at enrollment (on-site measurement) } & Mean \pm SD & $50.9 \pm 12.3$ & $51.5 \pm 11.6$ \\
\hline & $<50$ & $43(38.4)$ & $41(37.3)$ \\
\hline & 50 to $\leq 70$ & $69(61.6)$ & $68(61.8)$ \\
\hline \multirow[t]{4}{*}{ Anticoagulant therapy use } & Gabexate mesylate & $22(19.6)$ & $21(19.1)$ \\
\hline & Nafamostat mesylate & $6(5.4)$ & $4(3.6)$ \\
\hline & Thrombomodulin & $35(31.3)$ & $34(30.9)$ \\
\hline & Heparins & $31(27.7)$ & $32(29.1)$ \\
\hline \multirow[t]{2}{*}{ Replacement therapy use } & Fresh frozen plasma & $24(21.4)$ & $10(9.1)$ \\
\hline & Platelet concentrate & $26(23.2)$ & $22(20.0)$ \\
\hline Any anticoagulant use & & $83(74.1)$ & $76(69.1)$ \\
\hline
\end{tabular}

$n(\%), A P A C H E$ acute physiology and chronic health evaluation, $A T$ antithrombin, DIC disseminated intravascular coagulation, $p A T$ plasma-derived antithrombin, SOFA sequential organ failure assessment

(95\% CI 79.6-92.9\%) in the AT gamma group and $77.7 \%$ (95\% CI 68.8-85.0\%) in the pAT group (Table 3). The change in SOFA score on day 6 (or at withdrawal) was $-3.1 \pm 3.3$ in the AT gamma group and $-2.6 \pm 3.6$ in the pAT group. The change in APACHE II score on day 6 (or at withdrawal) was $-3.5 \pm 6.1$ in the AT gamma group and $-2.8 \pm 6.1$ in the pAT group. The change in plasma AT activity on day 6 (or at withdrawal) was $49.9 \% \pm 23.7 \%$ in the AT gamma group and $58.8 \% \pm$ $24.9 \%$ in the pAT group. The data regarding the changes of coagulation markers such as platelet count (PLT), fibrin/fibrinogen degradation products (FDP), and prothrombin time (PT) were also similar in both treatment groups. The mean \pm SD changes in PLT, FDP, and PT were $3.28 \pm 8.26 \times 10^{4} / \mu \mathrm{L}$ vs. $4.83 \pm 9.86 \times 10^{4} / \mu \mathrm{L}$, $138.65 \pm 649.01 \mu \mathrm{g} / \mathrm{mL}$ vs. $-111.81 \pm 536.59 \mu \mathrm{g} / \mathrm{mL}$, and $-2.65 \pm 5.94 \mathrm{~s}$ vs. $-2.08 \pm 3.85 \mathrm{~s}$, respectively, in the pAT and AT gamma groups.

\section{Safety}

The safety analysis set consisted of 221 patients (108 patients in the AT gamma group and 113 patients in the pAT group), after exclusion of one patient in the AT gamma group who withdrew before study treatment. There were no marked differences in the incidences of adverse events (AEs) and adverse drug 
Table 2 Primary endpoint: presence or absence of DIC recovery on day 6 (or at withdrawal)

\begin{tabular}{lll}
\hline AT activity (\%) & DIC recovery & \\
\cline { 2 - 3 } & pAT & AT gamma \\
& $\%(n / \mathrm{N})$ & $\%(n / \mathrm{N})$ \\
\hline$<50$ & $46.5(20 / 43)$ & $46.3(19 / 41)$ \\
$50-70$ & $56.5(39 / 69)$ & $61.8(42 / 68)$ \\
Total [95\% Cl] & $52.7(59 / 112)[43.0-62.2]$ & $56.4(62 / 110)[46.6-65.8]$ \\
Adjusted $^{\mathrm{a}}[95 \% \mathrm{Cl}]$ & $52.7[43.6-61.9]$ & $55.9[46.7-65.1]$ \\
\hline
\end{tabular}

$A T$ antithrombin, $\mathrm{Cl}$ confidence interval, $D / C$ disseminated intravascular coagulation, pAT plasma-derived antithrombin

${ }^{\text {a }}$ The protocol was amended to include all patients with AT activity $\leq 70 \%$ so that the patient population would more accurately reflect the intended clinical use. The (adjusted) DIC recovery rate and its $95 \% \mathrm{Cl}$ were calculated for each treatment group after stratification (using the Woolson-Bean method) by the AT activity $(<50 \%, 50-70 \%)$ at study enrollment

reactions (ADRs) (Table 4). AEs occurred in $89(82.4 \%)$ of 108 patients in the AT gamma group and 99 (87.6\%) of 113 patients in the pAT group. ADRs occurred in 24 patients $(22.2 \%)$ in the AT gamma group and 16 patients (14.2\%) in the pAT group. The most common AE was erythema in both the AT gamma and pAT groups, occurring in 18 patients (16.7\%) and 12 patients (10.6\%), respectively (Table 5).

AEs that led to death occurred in 10 (9.3\%) of the 108 patients in the AT gamma group and 22 (19.5\%) of
Table 3 Secondary endpoint: 28-day survival rate

\begin{tabular}{|c|c|c|}
\hline \multirow[t]{3}{*}{ AT activity (\%) } & \multicolumn{2}{|l|}{ Survival } \\
\hline & $\overline{\mathrm{pAT}}$ & AT gamma \\
\hline & $\%(n / N)$ & $\%(n / M)$ \\
\hline$<50$ & $76.7(33 / 43)$ & $85.4(35 / 41)$ \\
\hline $50-70$ & $78.3(54 / 69)$ & $89.7(61 / 68)$ \\
\hline Total $[95 \% \mathrm{Cl}]$ & $77.7(87 / 112)$ [68.8-85.0] & $87.3(96 / 110)$ [79.6-92.9] \\
\hline Adjusted ${ }^{\mathrm{a}}[95 \% \mathrm{Cl}]$ & $77.7[70.7-84.7]$ & $88.1[81.1-95.0]$ \\
\hline
\end{tabular}

$A T$ antithrombin, $\mathrm{Cl}$ confidence interval, $p A T$ plasma-derived antithrombin ${ }^{a}$ The protocol was amended to include all patients with AT activity $\leq 70 \%$ so that the patient population would more accurately reflect the intended clinical use. The (adjusted) survival rate and its $95 \% \mathrm{Cl}$ were calculated for each treatment group after stratification using the Woolson-Bean method, stratified by the AT activity $(<50 \%, 50-70 \%)$ at study enrollment

the 113 patients in the pAT group (Table 4). Of these, events that occurred in two patients (1.9\%) in the AT gamma group were classified as ADRs. The most common $\mathrm{AE}$ that led to death was sepsis, occurring in four patients (3.7\%) in the AT gamma group and seven patients $(6.2 \%)$ in the pAT group. In addition, other serious AEs occurred in $14(13.0 \%)$ of the 108 patients in the AT gamma group and seven (6.2\%) of the 113 patients in the pAT group. Of these, events for which a causal relationship to the investigational product was

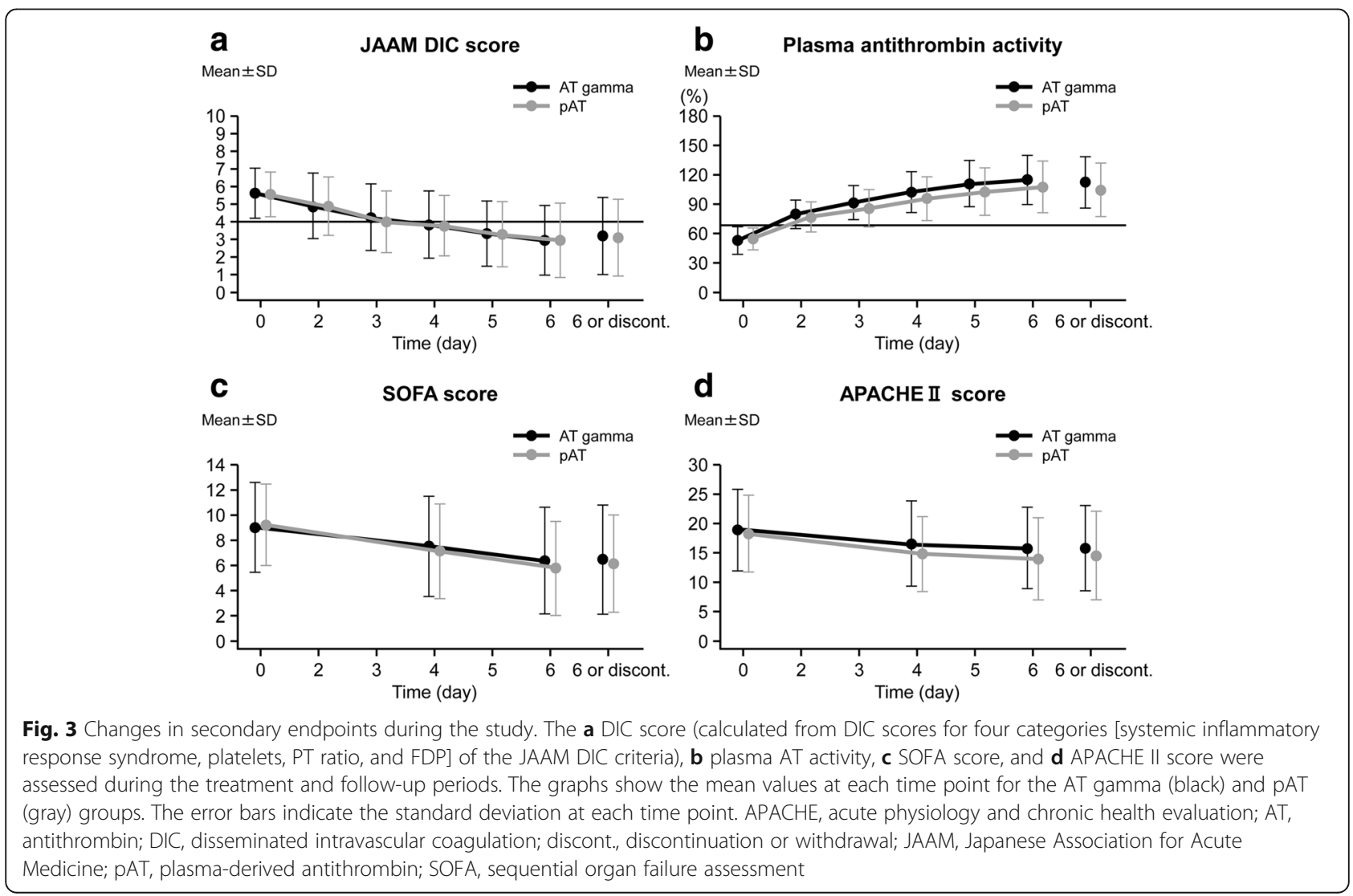


Table 4 Adverse events and adverse drug reactions

\begin{tabular}{cll}
\hline & pAT & AT gamma \\
& $N=113$ & $N=108$ \\
\hline AEs, $n(\%)$ & $99(87.6)$ & $89(82.4)$ \\
AEs that led to death & $22(19.5)$ & $10(9.3)$ \\
Other serious AEs & $7(6.2)$ & $14(13.0)$ \\
ADRs, $n(\%)$ & $16(14.2)$ & $24(22.2)$ \\
ADRs that led to death & $0(0)$ & $2(1.9)$ \\
Other serious ADRs & $1(0.9)$ & $3(2.8)$ \\
\hline
\end{tabular}

$A D R$ adverse drug reaction, $A E$ adverse event, $p A T$ plasma-derived antithrombin

not ruled out occurred in three patients $(2.8 \%)$ in the AT gamma group (i.e., gastrointestinal hemorrhage, cerebral infarction, and hemorrhagic transformation stroke) and one patient (0.9\%) in the pAT group (i.e., hemothorax). In the AT gamma group, there were 27 episodes of bleeding, and in the PAT group, there were 32. Apart from these, there were no other significant AEs.

In both the AT gamma and pAT groups, there were some patients whose laboratory values fluctuated during the study period or changed from baseline at the end-of-study examination. C-reactive protein levels, although they remained high, tended to decrease over time in both groups after the start of study treatment. However, for other test parameters, no consistent trend was observed. Transient changes in vital signs were observed in some patients in both groups, but no consistent trend was observed. All patients tested negative for antibodies to AT gamma at all time points.

\section{Discussion}

This multicenter, open-label, randomized, parallel-group study investigated the efficacy and safety of AT gamma in patients with sepsis-induced DIC. All efficacy endpoints were similar in patients receiving $36 \mathrm{IU} / \mathrm{kg} /$ day AT gamma and patients receiving $30 \mathrm{IU} / \mathrm{kg} /$ day $\mathrm{pAT}$. The protocol for this study was amended to include patients being treated with thrombomodulin and patients with AT activity below $50 \%$. This change did not affect the analysis of the primary endpoint because there was no difference in AT activity or thrombomodulin use between the two groups. The rate of heparin use was also similar in the two groups.

With the inclusion of patients with AT activity below $50 \%$, we were able to perform an analysis of the primary endpoint stratified by AT activity at enrollment $(<50 \%$ or $50-70 \%)$. The rate of DIC recovery was similar in the two treatment groups for patients with less than 50\% AT activity at baseline ( $46.3 \%$ vs. $46.5 \%$ for AT gamma and pAT, respectively). The DIC recovery rate was $61.8 \%$ in patients in the $50-70 \%$ AT activity group receiving AT gamma and $56.5 \%$ in patients receiving PAT, but no test of the significance of this difference was specified in the analysis plan. In

Table 5 Adverse events occurring in at least $5 \%$ of patients in either treatment group

\begin{tabular}{|c|c|c|c|c|}
\hline & \multicolumn{2}{|l|}{$\operatorname{pAT}(N=113)$} & \multicolumn{2}{|l|}{ AT gamma $(N=108)$} \\
\hline & Number of patients (\%) & Number of events & Number of patients (\%) & Number of events \\
\hline Erythema & $12(10.6)$ & 14 & $18(16.7)$ & 19 \\
\hline Diarrhea & $6(5.3)$ & 6 & $17(15.7)$ & 18 \\
\hline Decubitus ulcer & $10(8.8)$ & 12 & $12(11.1)$ & 13 \\
\hline Pneumonia & $6(5.3)$ & 6 & $10(9.3)$ & 10 \\
\hline Vomiting & $7(6.2)$ & 8 & $9(8.3)$ & 11 \\
\hline Constipation & $4(3.5)$ & 5 & $9(8.3)$ & 9 \\
\hline Insomnia & $10(8.8)$ & 10 & $9(8.3)$ & 9 \\
\hline Anemia & $8(7.1)$ & 9 & $7(6.5)$ & 7 \\
\hline Sepsis & $8(7.1)$ & 8 & $6(5.6)$ & 6 \\
\hline Hypokalemia & $9(8.0)$ & 9 & $5(4.6)$ & 7 \\
\hline Hypernatremia & $6(5.3)$ & 6 & $4(3.7)$ & 4 \\
\hline Pleural effusion & $8(7.1)$ & 8 & $4(3.7)$ & 4 \\
\hline Nausea & $7(6.2)$ & 7 & $3(2.8)$ & 4 \\
\hline Hepatic function abnormal & $6(5.3)$ & 6 & $3(2.8)$ & 3 \\
\hline Blood bilirubin increased & $6(5.3)$ & 6 & $3(2.8)$ & 3 \\
\hline Delirium & $6(5.3)$ & 6 & $3(2.8)$ & 3 \\
\hline Skin exfoliation & $11(9.7)$ & 17 & $3(2.8)$ & 3 \\
\hline
\end{tabular}

pAT plasma-derived antithrombin 
patients being treated with thrombomodulin, the DIC recovery rate was $64.7 \%$ in the AT gamma group and $40.0 \%$ in the pAT group. Survival rates on day 28 were $87.3 \%$ vs. 77.7\% for the AT gamma group and pAT group, respectively, regardless of AT activity at enrollment. The survival rate on day 28 was similar in patients not using thrombomodulin, but in patients using thrombomodulin, the rates were $94.1 \%$ vs. $71.4 \%$ in the AT gamma and pAT groups, respectively.

The safety of AT gamma was comparable to that of pAT in this study. The lists of AEs occurring in at least $5 \%$ of patients were similar in the AT gamma and pAT groups. Common AEs included erythema, diarrhea, and decubitus ulcer. The number of bleeding events was similar in the two groups, and bleeding was infrequent even with concomitant use of thrombomodulin and heparin. In a separate clinical study, we previously found that AT gamma is also effective for patients with DIC secondary to blood disorders or malignant tumors (unpublished observation, S. Endo, Morioka Yuai Hospital, Morioka, Japan).

AT gamma is a recombinant AT preparation produced in Chinese hamster ovary host cells. This novel preparation of AT has the same amino acid sequence and the same main type of oligosaccharides (complex-type nonfucosylated N-linked oligosaccharides) as native human AT and, therefore, also has similar biological activity. Because the existing AT preparation is derived from human blood, safety measures against infectious diseases, such as virus inactivation and removal, must be performed during the manufacturing process; however, the risk of transmission of an infectious agent cannot be completely excluded. Furthermore, plasma-derived products are dependent on blood donations, and the blood donation population is not guaranteed. Because AT gamma is a genetically modified preparation, there is no risk of infection and the supply can be stably maintained.

Although recombinant AT alfa has been approved for use in patients with congenital AT deficiency, its half-life is markedly shorter than the half-life of native AT, and it must be administered continuously for $24 \mathrm{~h}$. In contrast, AT gamma has been developed with a similar glycosylation profile to native AT, and its longer half-life than AT alfa allows for a short, 1-h infusion. We expect that the 1-h infusion will be more convenient in the hospital.

Our study has some limitations that should also be considered. First, the study was conducted open-label and was not designed to statistically compare the two treatment groups. Second, the study enrolled only patients with DIC with underlying infectious disease. Patients with other causes of DIC might respond differently to AT gamma. Third, long-term outcomes beyond 28 days were not assessed. Lastly, approximately half of the patients in the study were being treated with other anticoagulation therapies during the study.

\section{Conclusions}

The results of this study demonstrate that $36 \mathrm{IU} / \mathrm{kg} /$ day of AT gamma is as efficacious and safe as $30 \mathrm{IU} / \mathrm{kg} /$ day of pAT. Thus, this study establishes AT gamma as an alternative to $\mathrm{pAT}$ for addressing this critical clinical need.

\section{Abbreviations}

ADR: Adverse drug reaction; AE: Adverse event; APACHE: Acute physiology and chronic health evaluation; AT: Antithrombin; Cl: Confidence interval; DIC: Disseminated intravascular coagulation (syndrome); FDP: Fibrin/ fibrinogen degradation products; PAT: Plasma-derived antithrombin; PLT: Platelet count; PT: Prothrombin time; SOFA: Sequential organ failure assessment

\section{Acknowledgements}

We would like to thank all investigators and centers participating in this study as part of the Antithrombin Gamma Study Group. The investigators and their centers were as follows: Satoshi Gando (Emergency and Critical Care Center, Hokkaido University Hospital); Shigeto Oda (Department of Emergency and Critical Care Medicine, Chiba University Hospital); Yasuhiro Otomo (Trauma and Acute Critical Care Medical Center, Tokyo Medical and Dental University, Medical Hospital); Masanao Miura (Department of Emergency and Critical Care Medicine, Kariya Toyota General Hospital); Shinji Ogura (Advanced Critical Care Center, Gifu University Hospital); Yutaka Eguchi (Department of Critical and Intensive Care Medicine, Shiga University of Medical Science Hospital); Joji Kotani (Emergency and Critical Care Center, Hyogo College of Medicine); Norio Yamashita (Advanced Emergency Medical Service Center, Kurume University Hospital); Hiroyasu Ishikura (Emergency and Critical Care Center, Fukuoka University Hospital); Yuichiro Sakamoto (Emergency and Critical Care Center, Saga University Hospital); Takeshi Takahashi (Department of Emergency and Critical Care Medicine, National Hospital Organization Kumamoto Medical Center); Yasushi Suzuki (Iwate Prefectural Advanced Critical Care and Emergency Center, Iwate Medical University Hospital); Shigeki Kushimoto (Division of Emergency and Critical Care Medicine, Tohoku University Hospital); Nobuya Kitamura (Department of Emergency and Critical Care Medicine, Kimitsu-Chuo Hospital); Naoyuki Matsuda (Department of Emergency and Critical Care Medicine, Nagoya University Hospital); Naohiro Fujita (Emergency and Critical Care Center, Saga Prefectural Hospital Koseikan); Mitsuhide Kitano (Emergency and Critical Care Center, Saiseikai Yokohamashi Tobu Hospital); Junko Yamaguchi (Emergency and Critical Care Center, Nihon University Itabashi Hospital); Yoshikazu Yasuda (Department of Intensive Care Unit, University of Fukui Hospital); Hayato Takayama (Emergency and Intensive Care Center, National Hospital Organization Nagasaki Medical Center); Toshiharu Tanaka (Emergency and Critical Care Medical Center, Niigata City General Hospital); Tetsuya Matsuoka (Osaka Prefectural Senshu Critical Care Medical Center, Rinku General Medical Center); Tetsuhiro Takei (Department of Emergency and Critical Care Medicine, Yokohama City Minato Red Cross Hospital); Tsuyoshi Hatada (Emergency Critical Care Center, Mie University Hospital); Masahiro Tamashiro (Department of Cardiology, Tomishiro Central Hospital); Satoshi Fujimi (Critical Care and Trauma Center, Osaka General Medical Center); Osamu Nishida (Department of Anesthesiology and Critical Care Medicine, Fujita Health University Hospital); Kazuhito Tamehiro (Department of Emergency Medicine, St. Mary's Hospital); Junichi Maehara (Department of Acute Care and General Medicine, Saiseikai Kumamoto Hospital); Shinsuke Fujiwara (Emergency Medicine, National Hospital Organization Ureshino Medical Center); and Hideo Wada (Department of Molecular Laboratory Medicine, Mie University Graduate School of Medicine). We also thank Susan Cottrell, PhD, of Edanz Medical Writing for providing medical writing support.

\section{Funding}

AT gamma is being developed by Kyowa Hakko Kirin Co., Ltd. This study was sponsored by Kyowa Hakko Kirin Co., Ltd., which had a role in the design of the study and in the collection, analysis, and interpretation of the data.

\section{Availability of data and materials}

The datasets generated during the current study are not publicly available and remain proprietary. 


\section{Authors' contributions}

Both authors participated in the conception and design of the study. Both authors were involved in the analysis and interpretation of the data. SE drafted the manuscript. SE and RS provided intellectual content of critical importance. Both authors revised and approved the final version of the manuscript. The Antithrombin Gamma Study Group contributed to the implementation and completion of this study, and the names and affiliations of individuals in the group are detailed in the "Acknowledgements."

\section{Ethics approval and consent to participate}

This study was approved by all local ethics committees at participating institutions. All patients provided written informed consent. The protocol was approved by the institutional review boards of all participating institutes (see "Acknowledgements").

\section{Consent for publication}

Not applicable.

\section{Competing interests}

SE has received consulting/lecture fees and research funding from Kyowa

Hakko Kirin Co., Ltd. RS is employed by Kyowa Hakko Kirin Co., Ltd.

\section{Publisher's Note}

Springer Nature remains neutral with regard to jurisdictional claims in published maps and institutional affiliations.

\section{Author details}

${ }^{1}$ Morioka Yuai Hospital, Nagai 12-10, Morioka, Iwate 020-0834, Japan. ${ }^{2}$ R\&D

Division, Kyowa Hakko Kirin Co., Ltd., Tokyo, Japan.

Received: 13 April 2018 Accepted: 3 October 2018

Published online: 16 November 2018

\section{References}

1. Koide T. Isolation and characterization of antithrombin III from human, porcine and rabbit plasma, and rat serum. J Biochem. 1979;86:1841-50.

2. Nordenman B, Nystrom C, Bjork I. The size and shape of human and bovine antithrombin III. Eur J Biochem. 1977;78:195-203.

3. Damus PS, Hicks M, Rosenberg RD. Anticoagulant action of heparin. Nature. 1973;246:355-7.

4. Olson ST, Bjork I. Regulation of thrombin activity by antithrombin and heparin. Semin Thromb Hemost. 1994;20:373-409.

5. Jin L, Abrahams JP, Skinner R, Petitou M, Pike RN, Carrell RW. The anticoagulant activation of antithrombin by heparin. Proc Natl Acad Sci U S A. 1997:94: 14683-8.

6. Gando S, Levi M, Toh CH. Disseminated intravascular coagulation. Nat Rev Dis Primers. 2016;2:16037.

7. Baglin T. Disseminated intravascular coagulation: diagnosis and treatment. BMJ. 1996;312:683-7.

8. Wada H, Asakura H, Okamoto K, Iba T, Uchiyama T, Kawasugi K, Koga S, Mayumi T, Koike K, Gando S, Kushimoto S, Seki Y, Madoiwa S, Maruyama I, Yoshioka A. Expert consensus for the treatment of disseminated intravascular coagulation in Japan. Thromb Res. 2010;125:6-11.

9. Warren BL, Eid A, Singer P, Pillay SS, Carl P, Novak I, Chalupa P, Atherstone A, Penzes I, Kubler A, Knaub S, Keinecke HO, Heinrichs H, Schindel F, Juers M, Bone RC, Opal SM. Caring for the critically ill patient. High-dose antithrombin III in severe sepsis: a randomized controlled trial. JAMA. 2001;286:1869-78.

10. Kienast J, Juers M, Wiedermann CJ, Hoffmann JN, Ostermann H, Strauss R, Keinecke HO, Warren BL, Opal SM. Treatment effects of high-dose antithrombin without concomitant heparin in patients with severe sepsis with or without disseminated intravascular coagulation. J Thromb Haemost. 2006:4:90-7.

11. Wiedermann CJ, Kaneider NC. A systematic review of antithrombin concentrate use in patients with disseminated intravascular coagulation of severe sepsis. Blood Coagul Fibrinolysis. 2006;17:521-6.

12. Hayakawa M, Kudo D, Saito S, Uchino S, Yamakawa K, lizuka Y, Sanui M, Takimoto K, Mayumi T, Ono K, Azuhata T, Ito F, Yoshihiro S, Hayakawa K, Nakashima T, Ogura T, Noda E, Nakamura Y, Sekine R, Yoshikawa Y, Sekino M, Ueno K, Okuda Y, Watanabe M, Tampo A, Saito N, Kitai Y, Takahashi H, Kobayashi I, Kondo Y, Matsunaga W, Nachi S, Miike T, Takahashi H, Takauji S, Umakoshi K, Todaka T, Kodaira H, Andoh K, Kasai T, Iwashita Y, Arai H, Murata M, Yamane M, Shiga K, Hori N. Antithrombin supplementation and mortality in sepsis-induced disseminated intravascular coagulation: a multicenter retrospective observational study. Shock. 2016:46:623-31.

13. Nishida O, Yuji O, Inoue S, Rika T, Imaizumi ES, Yasuyuki K. The Japanese Clinical Practice Guidelines for management of sepsis and septic shock. J Japanese Assoc Acute Med. 2016;2016:28.

14. Paidas MJ, Forsyth C, Quere I, Rodger M, Frieling JT, Tait RC. Perioperative and peripartum prevention of venous thromboembolism in patients with hereditary antithrombin deficiency using recombinant antithrombin therapy. Blood Coagul Fibrinolysis. 2014;25:444-50.

15. Edmunds T, Van Patten SM, Pollock J, Hanson E, Bernasconi R, Higgins E, Manavalan P, Ziomek C, Meade H, McPherson JM, Cole ES. Transgenically produced human antithrombin: structural and functional comparison to human plasma-derived antithrombin. Blood. 1998;91:4561-71.

16. Matsushita T. Engineered therapeutic antibodies with enhanced effector functions: clinical application of the Potelligent ${ }^{\circledR}$ technology. Korean J Hematol. 2011;46:148-50.

17. Gando S, Iba T, Eguchi Y, Ohtomo Y, Okamoto K, Koseki K, Mayumi T, Murata A, Ikeda T, Ishikura H, Ueyama M, Ogura H, Kushimoto S, Saitoh D, Endo S, Shimazaki S. A multicenter, prospective validation of disseminated intravascular coagulation diagnostic criteria for critically ill patients: comparing current criteria. Crit Care Med. 2006;34:625-31.

\section{Ready to submit your research? Choose BMC and benefit from:}

- fast, convenient online submission

- thorough peer review by experienced researchers in your field

- rapid publication on acceptance

- support for research data, including large and complex data types

- gold Open Access which fosters wider collaboration and increased citations

- maximum visibility for your research: over $100 \mathrm{M}$ website views per year

At $\mathrm{BMC}$, research is always in progress.

Learn more biomedcentral.com/submissions 